\title{
The dynamics of the massless scalar field coupled to LQG in the polymer quantization
}

\author{
Jerzy Lewandowski* \\ Institute of Theoretical Physics, Warsaw University, ul. Hoża 69, 00-681 Warsaw, Poland \\ Institute for Quantum Gravity (IQG), FAU Erlangen - Nürnberg, Staudtstr. 7, 91058 Erlangen, \\ Germany \\ E-mail: Jerzy. Lewandowski@fuw.edu.pl
}

\section{Marcin Domagała}

Institute of Theoretical Physics, Warsaw University, ul. Hoża 69, 00-681 Warsaw, Poland

E-mail: Marcin.Domagala@fuw.edu.pl

\section{Michał Dziendzikowski}

Institute of Theoretical Physics, Warsaw University, ul. Hoża 69, 00-681 Warsaw, Poland

E-mail: Michal.Dziendzikowski@fuw.edu.pl

\begin{abstract}
The polymer quantization of matter fields is a diffeomorphism invariant framework compatible with Loop Quantum Gravity. While studied by itself, it is not explicitly used in the known completely quantizable models of matter coupled to LQG. In the current paper we apply the polymer quantization to the model of massless scalar field coupled to LQG. We show that the polymer Hilbert space of the field degrees of freedom times the LQG Hilbert space of the geometry degrees of freedom admit the quantum constraints of GR and accommodate their explicit solutions. In this way the quantization can be completed. That explicit way of solving the quantum constraints suggests interesting new ideas.
\end{abstract}

3rd Quantum Gravity and Quantum Geometry School,

February 28 - March 13, 2011

Zakopane, Poland

\footnotetext{
* Speaker.
} 


\section{Introduction}

\subsection{Our goal}

A successful quantization of the gravitational field does not complete the standard model of fundamental interactions. All the standard matter fields need to be quantized in a compatible way. In particular, the standard Fock space quantization is not available. In Loop Quantum Gravity [1, 2, $3,4]$ a new diffeomorphism invariant framework for quantum matter field operators was introduced. In particular, the scalar field is quantized according to the polymer quantization $[5,6,7,8,9]$. On the other hand, more recent quantum models of matter interacting with the quantum geometry of LQG seem not to need any specific quantization of a scalar field itself $[10,11,12,13,14]$. For example, when the scalar constraint of General Relativity is solved classically, it swallows one scalar field which effectively becomes a parameter labeling the observables. Therefore, this scalar field is treated in a different way, than other fields. Another insight comes from the Loop Quantum Cosmology. Within that framework, whereas the homogeneous gravitational degrees of freedom are polymer quantized, the homogeneous scalar field is quantized in a standard Quantum Mechanics fashion. Hence, the framework is inconsistent in the way the scalar field is quantized as opposed to the gravitational field. A third example is the full LQG model of the massless scalar field coupled to gravity [13]. The final formulation of the model is exact and precise, the Hilbert space and the quantum physical hamiltonian are clearly defined modulo the issue of the self-adjoint extensions which is not addressed. However, the derivation that leads to that result assumes the existence of a suitable quantization of the scalar which is not used explicitly.

The goal of our current work is to show, that the polymer quantization of matter fields can be used for coupling them with LQG. We demonstrate it on two known examples of massless scalar field coupled to gravity: (i) a warming up example is the homogeneous isotropic model of Loop Quantum Cosmology $[15,16]$, and (ii) the main example is the case with all the local degrees of freedom of the full Loop Quantum Gravity [13].

\subsection{The Polymer quantization}

We recall here the Polymer quantization. Consider an $n$-dimensional real manifold $\Sigma$ (a 3D Cauchy surface in the case of GR), a real valued scalar field $\varphi: \Sigma \rightarrow \mathbb{R}$, the canonically conjugate momentum $\pi$, and the Poisson bracket

$$
\{\varphi(x), \pi(y)\}=\delta(x, y), \quad\{\varphi(x), \varphi(y)\}=0=\{\pi(x), \pi(y)\} .
$$

Notice, that $\pi$ is a density of weight 1 , that is, upon a change of coordinates $\left(x^{a}\right) \mapsto\left(x^{\prime a}\right)$ it transforms as a measure, that is

$$
\pi(x) d^{n} x=\pi\left(x^{\prime}\right) d^{n} x^{\prime} .
$$

A Polymer variable representing $\pi$ is defined for every open, finite $\mathscr{U} \subset \Sigma$,

$$
\pi(V)=\int_{V} d^{n} x \pi(x) .
$$

A Polymer variable $U_{p}$ representing $\varphi$ is assigned to every function $p: \Sigma \rightarrow \mathbb{R}$

$$
x \mapsto p_{x}
$$


of a finite support,

$$
U_{p}(\varphi)=e^{i \sum_{x \in \Sigma} p_{x} \varphi(x)}
$$

In particular

$$
U_{p=0}(\varphi)=1
$$

Notice, that

$$
\operatorname{supp} p=\left\{x_{1}, \ldots, x_{n}\right\}, \quad U_{p}(\varphi)=e^{i\left(p_{x_{1}} \varphi\left(x_{1}\right)+\ldots+p_{x_{n}} \varphi\left(x_{n}\right)\right)}
$$

The Poisson bracket between the Polymer variables is

$$
\left\{U_{\pi}, \pi(V)\right\}=i\left(\sum_{x \in V} p_{x}\right) U_{p}, \quad\left\{U_{p}, U_{p^{\prime}}\right\}=0=\left\{\pi(V), \pi\left(V^{\prime}\right)\right\} .
$$

The Polymer quantization consists in using the following vector space

$$
\left\{a_{1} U_{p_{1}}+\ldots+a_{k} U_{p_{k}}: a_{I} \in \mathbb{C}, k \in \mathbb{N}\right\}
$$

endowed with the following Hilbert product

$$
\left(U_{p} \mid U_{p^{\prime}}\right)=\delta_{p, p^{\prime}}
$$

where the Kronecker delta takes values 0 or 1 . That is we introduce the Hilbert space

$$
\mathscr{H}:=\overline{\left\{a_{1} U_{p_{1}}+\ldots+a_{k} U_{p_{k}}: a_{I} \in \mathbb{C}, k \in \mathbb{N}\right\}} .
$$

Considered as an element of $\mathscr{H}$, the function $U_{p}$ will be denoted by

$$
U_{p}=:|p\rangle \text {. }
$$

The Polymer variables give rise to the Polymer operators

$$
\hat{U}_{p}\left|p^{\prime}\right\rangle=\left|p+p^{\prime}\right\rangle, \quad \hat{\pi}(V)|p\rangle=\hbar\left(\sum_{x \in V} p_{x}\right)|p\rangle
$$

Hence, the values $p_{x}$ taken by the function $p$ account to the spectrum of the $\hat{\pi}(V)$ operators. For this reason, in the quantum context we will modify the notation and write

$$
\hat{\pi}(V)|\pi\rangle=\hbar\left(\sum_{x \in \Sigma} \pi_{x}\right)|\pi\rangle, \quad \hat{U}_{\pi}\left|\pi^{\prime}\right\rangle=\left|\pi+\pi^{\prime}\right\rangle
$$

denoting by $\pi$ and $\pi^{\prime}$ functions of the finite support

$$
x \mapsto \pi_{x}, \pi_{x}^{\prime} \in \mathbb{R} .
$$

The advantage of the polymer quantization is that the diffeomorphism of $\Sigma$ act naturally as unitary operators in the Hilbert space. This is what makes this quantization different from the standard one. 
Remark Diffeomorphism invariant quantizations of the Polymer variables were studied in [8, 9] and a class of inequivalent quantizations parametrized by a real parameter a was found:

$$
\hat{\pi}(V)|\pi\rangle=\hbar\left(\sum_{x \in V} \pi_{x}+a \mathrm{E}(V)\right)|\pi\rangle
$$

where $\mathrm{E}(V)$ is the Euler characteristics of $V$ and $\hat{U}_{\pi}$ is the same as above, independently on the value of $a$. However, nobody has ever used any of them for $a \neq 0$.

There is also a 1-degree of freedom "poor man" version of the Polymer quantization that can be applied to mechanics. Consider a variable $\Phi \in \mathbb{R}$ and the conjugate momentum $\Pi$, and the Poisson bracket defined by

$$
\{\Phi, \Pi\}=1 \quad\{\Phi, \Phi\}=0=\{\Pi, \Pi\} .
$$

The Polymer variables are $\Pi$ itself, and for every $p \in \mathbb{R}$,

$$
\tilde{U}_{\pi}(\Phi):=e^{i p \Phi} .
$$

The Polymer quantum representation of those variables is defined in the seemingly usual way

$$
\hat{U}_{\pi} \psi(\Phi)=\tilde{U}_{\pi}(\Phi) \psi(\Phi), \quad \hat{\Pi} \psi(\Phi)=\frac{\hbar}{i} \frac{d}{d \Phi} \psi(\Phi),
$$

in an unusual Hilbert space, though

$$
\tilde{\mathscr{H}}:=\overline{\left\{a_{1} \tilde{U}_{\pi_{1}}+\ldots+a_{k} \tilde{U}_{\pi_{k}}: a_{I} \in \mathbb{C}, k \in \mathbb{N}, \pi_{I} \in \mathbb{R}\right\}}
$$

with the Hilbert product defined such that the $U_{\pi}$ functions are normalizable

$$
\left(\tilde{U}_{\pi} \mid \tilde{U}_{\pi^{\prime}}\right)=\delta_{\pi, \pi^{\prime}}
$$

If we again denote

$$
\tilde{U}_{\pi}=:|\pi\rangle,
$$

whenever it is considered an element of $\tilde{\mathscr{H}}$, then

$$
\hat{\tilde{U}}_{\pi}\left|\pi^{\prime}\right\rangle=\left|\pi+\pi^{\prime}\right\rangle, \quad \hat{\Pi}|\pi\rangle=\hbar \pi|\pi\rangle .
$$

Actually, even a polymer quantum mechanics was considered in the literature $[17,18,19]$.

The polymer quantization Hilbert spaces $\mathscr{H}$ and, respectively, $\tilde{\mathscr{H}}$ can be obtained by suitable integrals. The poor man Hilbert product can be defined by the Bohr measure such that

$$
\int_{\mathrm{R}_{\mathrm{Bohr}}} d \mu_{\mathrm{Bohr}}(\Phi) e^{i \pi \Phi}=\delta_{0, \pi}
$$

where $\widehat{B}_{\text {Bohr }}$ stands for the Bohr compactification of the line. With certain abuse of notation we often write

$$
\tilde{\mathscr{H}}=L_{2}(\overbrace{\text { Bohr }}) \text {. }
$$

In the scalar field case, the polymer Hilbert product is defined by the infinite tensor product of the Bohr measures, that is the natural Haar measure defined on the group $\widehat{R}_{\text {Bohr }}^{\Sigma}$ of all the maps $\Sigma \rightarrow{ }_{\text {Bohr. So one can write }}$

$$
\mathscr{H}=L_{2}\left({\stackrel{\left(\mathbb{R}_{\mathrm{Bohr}}\right.}{ }}^{\Sigma}\right) .
$$




\section{A doubly Polymer Quantization of LQC.}

A homogeneous and isotropic spacetime coupled to a KG scalar field is described by two real valued dynamical variables $c, \Phi$, and their conjugate momenta $p_{c}, \Pi$. The Poisson bracket $\{\cdot, \cdot\}$ is defined by

$$
\{\Phi, \Pi\}=1=\left\{c, p_{c}\right\},
$$

whereas the remaining brackets vanish. The first variable, $\Phi$, is the scalar field constant on the homogeneous 3-manifold $\Sigma$. The canonically conjugate variable $\Pi$ is defined by a suitable integral of the momentum $\pi$, also constant on $\Sigma$ by the homogeneity assumption. The variable $p$ is proportional to the square of the scale of the universe $\left(a^{2}\right)$, and $c$ to the rate of change in time $(\dot{a})$.

The constraints of General Relativity reduce to a single constraint, the Scalar Constraint - and the Hamiltonian of the system - which for a massless scalar field takes the following form [20]

$$
C_{ \pm}=\Pi \mp h\left(c, p_{c}\right),
$$

where $h$ is by definition a positive definite expression (this is the reduction of the familiar $\sqrt{-2 \sqrt{\operatorname{detq}} C_{\mathrm{gr}}}$ to the homogeneous isotropic gravitational fields).

According to the Wheeler de Witt quantization of this model (which was historically the first available), the both degrees of freedom are quantized in the usual way, that is the Hilbert space of the kinematical quantum states of the model is

$$
L_{2}(\mathbb{R}) \otimes L_{2}(\mathbb{R}) .
$$

The LQC quantization uses the holonomy variables of Loop Quantum Gravity restricted to the homogeneous isotropic solutions. The consequence is that the gravitational degree of freedom $c$ ends up quantized in the Polymer way [22]. The scalar field, on the other hand, is quantized in the usual way. Finally, the resulting Hilbert space of the kinematical quantum states of LQC is the hybrid Hilbert space

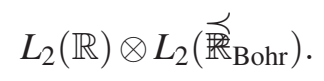

Those details were set in this way without deeper thinking, just because it works.

The goal of this section is to present a fully Polymer formulation of this LQC model in which the both variables $c$ and $\Phi$ are quantized in the Polymer way in the kinematical Hilbert space

$$
\mathscr{H}_{\text {kin }}=L_{2}\left(\widehat{\mathbb{R}_{\mathrm{Bohr}}}\right) \otimes L_{2}(\widehat{\widehat{R}} \text { Bohr })=: \mathscr{H}_{\text {mat }} \otimes \mathscr{H}_{\text {gr }} \text {. }
$$

Let $\hat{\Pi}$ be the operator defined according to 1.19 in the first factor $\mathscr{H}_{\text {mat }}$ polymer Hilbert space and let $\hat{h}$ be a quantum operator defined by a quantization of the term $h(x, p)$ in the second factor $\mathscr{H}_{\text {gr }}$ Polymer Hilbert space. Specifically, one can think of the operator defined in [20], or one of the wider class of operators considered in [21]. In fact, the operator is defined only in a suitable subspace of

$$
\mathscr{H}_{\mathrm{gr}, h} \subset \mathscr{H}_{\mathrm{gr}},
$$

because it involves square roots of other operators which are not positive definite, and only the positive parts of their spectra are physical. What will be important in this section is that $\hat{h}$ is selfadjoint (it is also non-negative) in $\tilde{\mathscr{H}}_{\mathrm{gr}, h}$. We will also have to reduce the full kinematical Hilbert 
space to

$$
\tilde{\mathscr{H}}_{\mathrm{kin}, h}=\mathscr{H}_{\text {mat }} \otimes \mathscr{H}_{\mathrm{gr}, h}
$$

The quantum constrain operator is

$$
\hat{C}_{ \pm}=\hat{\Pi} \otimes \mathrm{id} \mp \mathrm{id} \otimes \hat{h} .
$$

The Hilbert space of the physical states is spanned by the two spaces

$$
\mathscr{H}_{\text {phys } \pm}=\mathscr{H}_{\hat{C}_{ \pm}=0}
$$

of the spectral decompositions of the operators $\hat{C}_{ \pm}$corresponding to 0 in the spectrum of $\hat{C}_{+}$(respectively $\hat{C}_{-}$). As we will see below, that space consists of normalizable elements of (2.3).

The main device we use is an operator $e^{i \hat{\Phi} \otimes \hat{h}}$. Itself, an operator $\hat{\Phi}$ is not defined in the polymer Hilbert space, but the definition of $e^{i \hat{\Phi} \otimes \hat{h}}$ is quite natural if we use eigenvectors of the operator $\hat{h}$ : $\left\{\psi_{l}: l \in L\right\}$, where $L$ is a label set. In $\mathscr{H}_{\text {kin }}$ we consider the simultaneous eigenvectors of id $\otimes \hat{h}$ and $\hat{\Pi} \otimes \mathrm{id}$, that is

$$
\left\{|\pi\rangle \otimes \psi_{l}: \pi \in \mathbb{R}, l \in L\right\}
$$

Define

$$
e^{i \hat{\Phi} \otimes \hat{h}}|\pi\rangle \otimes \psi_{l}:=e^{i h_{l} \hat{\Phi}} \otimes \mathrm{id}|\pi\rangle \otimes \psi_{l}=\left|\pi+h_{l}\right\rangle \otimes \psi_{l} .
$$

This operator preserves the norm, and admits inverse, namely

$$
e^{-i \hat{\Phi} \otimes \hat{h}}|\pi\rangle \otimes \psi_{l}:=e^{-i h_{l} \hat{\Phi}} \otimes \mathrm{id}|\pi\rangle \otimes \psi_{l}=\left|\pi-h_{l}\right\rangle \otimes \psi_{l} .
$$

Therefore, it is a unitary operator in $\mathscr{H}_{\text {kin }, h}$.

The next step in the derivation of the physical states, their Hilbert space, and the Dirac observables is to notice that

$$
\hat{C}_{ \pm}=\hat{\Pi} \mp \hat{h}=e^{ \pm i \hat{\Phi} \otimes \hat{h}} \hat{\Pi} e^{\mp i \hat{\Phi} \otimes \hat{h}}
$$

For clarity, let us fix a sign in $\hat{C}_{ \pm}$and consider first, say, $\hat{C}_{+}$. Indeed, it follows that the spectral decomposition of $\hat{C}_{+}$is obtained from the spectral decomposition of $\hat{\Pi}$. In particular, the Hilbert space corresponding to 0 in the spectrum of $\hat{C}_{+}$is obtained from the Hilbert space of the decomposition of $\hat{\Pi} \otimes$ id corresponding to 0 in the spectrum, that is

$$
\mathscr{H}_{\text {phys }+}=e^{i \hat{\Phi} \otimes \hat{h}}\left(|0\rangle \otimes \mathscr{H}_{\text {gr }, \mathrm{h}}\right) \subset \mathscr{H}_{\text {kin }, h} .
$$

Secondly, it follows that

$$
\left[\hat{\mathscr{O}}, \hat{C}_{+}\right]=0 \Leftrightarrow\left[e^{-i \hat{\Phi} \otimes \hat{h}} \hat{\mathscr{O}} e^{i \hat{\Phi} \otimes \hat{h}}, \hat{\Pi} \otimes \mathrm{id}\right]=0 .
$$

The general solution for a Dirac observable is a function of the following basic solutions

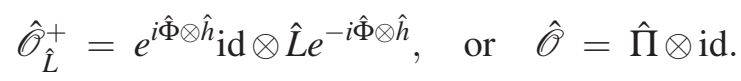

The second option above, however, on $\mathscr{H}_{\text {phys }+ \text { reduces to }}$

$$
\hat{\Pi} \otimes \mathrm{id}=\mathrm{id} \otimes \hat{h}=\hat{\mathscr{O}}_{\hat{h}}^{+} .
$$




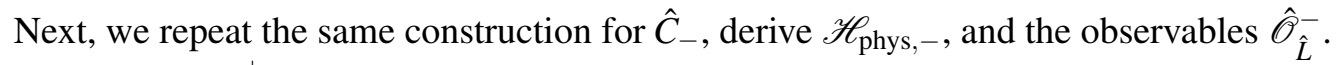

The spaces $\mathscr{H}_{\text {phys }}^{ \pm}$correspond to the non-negative/non-positive eigenvalues of the scalar field momentum $\hat{\Pi}$. They span a subspace

$$
\mathscr{H}_{\text {phys }} \subset \mathscr{H}_{\text {kin }} \text {. }
$$

If $\hat{h}$ is bounded from zero (for example for negative cosmological constant), then

$$
\mathscr{H}_{\text {phys }}=\mathscr{H}_{\text {phys }} \oplus \mathscr{H}_{\text {phys }-} \text {. }
$$

Otherwise, $\mathscr{H}_{\text {phys }} \cap \mathscr{H}_{\text {phys- }}$ is the subspace of states $|\pi=0\rangle \otimes\left|h_{l}=0\right\rangle$. In both cases, the observables $\hat{\mathscr{O}}_{\hat{L}}^{+}$and $\hat{\mathscr{O}}_{\hat{L}}^{-}$are consistent on the overlap and give rise to observables defined on $\mathscr{H}_{\text {phys }}$,

$$
\left.\hat{\mathscr{O}}_{\hat{L}}\right|_{\mathscr{H}_{\text {phys }, \pm}}=\hat{\mathscr{O}}_{\hat{L}}^{ \pm} .
$$

This result agrees with the known in the literature LQC model constructed by the hybrid quantization, but it is quantized by applying consequently the Polymer quantization to the both matter and gravity. This result generalizes in the obvious way to the homogeneous non-isotropic models, because the Hilbert space of the scalar field is insensitive on that generalization.

\section{The polymer quantization of LQG}

We turn now to the main subject of this work, the scalar field coupled to the gravitational field. This section should be read as a continuation of the lecture notes „From Classical To Quantum Gravity: Introduction to Loop Quantum Gravity " by Hanno Sahlmann and Kristina Giesel [23], another part of the current proceedings.

The canonical field variables are defined on a 3-manifold $\Sigma$. They are the scalar field $\varphi$ and its momentum $\pi$ introduced above in Section 1.2, and the Ashtekar-Barbero variables $A_{a}^{i}$ and $E_{b}^{j}$.

The kinematical Hilbert space for the quantum scalar field (1.8) will be denoted here by $\mathscr{H}_{\text {kin,mat }}$. The kinematical Hilbert space for the quantum gravitational field introduced in Section 3.1 of [23] out of the cylindrical functions of the variable $A$ (connection), will be denoted here by $\mathscr{H}_{\text {kin,gr }}$. The kinematical Hilbert space for the system is

$$
\mathscr{H}_{\text {kin }}=\mathscr{H}_{\text {kin,mat }} \otimes \mathscr{H}_{\text {kin,gr }},
$$

and its elements are functions

$$
(\varphi, A) \mapsto \psi(\varphi, A)
$$

\subsection{The Yang-Mills gauge transformations and the Gauss constraint}

Classically, the theory is constrained by the first class constraints: the Gauss constraint, the vector constraint and the scalar constraint.

The quantum Gauss constraint operator $\operatorname{id} \otimes \hat{\mathscr{G}}(\Lambda)$, acts on the gravitational degrees of freedom where the operator $\hat{\mathscr{G}}(\Lambda)$ is defined for every $\Lambda: \Sigma \rightarrow \mathfrak{s u}(2)$ in Section 3.2.1 of [23]. The operator induces the unitary group of the "Yang-Mills gauge transformations" acting in $\mathscr{H}_{\text {kin,gr }}$,

$$
\psi \mapsto U^{\mathrm{G}}(a) \psi, \quad U^{\mathrm{G}}(a) \psi(\varphi, A)=\psi\left(\varphi, a^{-1} A a+a^{-1} d a\right) .
$$


The space of solutions to the Gauss constraint in $\mathscr{H}_{\text {kin,gr }}$ was characterized at the end of Section 3.3.1 in [23] (and denoted by $\mathscr{H}_{\text {kin }}^{G}$ ). In the current paper, we will be denoting it by $\mathscr{H}_{\text {kin,gr }}^{G}$. The space of the solutions to the quantum Gauss constraint in $\mathscr{H}_{\text {kin }}$ is

$$
\mathscr{H}_{\text {kin }}^{G}=\mathscr{H}_{\text {kin,mat }} \otimes \mathscr{H}_{\text {kin,gr }}^{G}
$$

This is a subspace of $\mathscr{H}_{\text {kin }}$ which consists of the elements invariant with respect to the Yang-Mills gauge transformations. In terms of the generalized spin-networks, this subspace is the completion of the span of the subspaces $\mathscr{H}_{\gamma, \vec{j}, \vec{l}=0}$. There is an equivalent constructive definition of the solutions called the group averaging. It consists in integration with respect to the gauge transformations

$$
\psi \mapsto \int \prod_{x \in \Sigma} d a(x) \psi\left(\varphi, a^{-1} A a+a^{-1} d a\right)
$$

This kind of integral usually would be defined only "formally". However, if $A \mapsto \psi(\varphi, A)$ is a function cylindrical with respect to a graph $\gamma$ embedded in $\Sigma$, then the Yang-Mills gauge transformations act at the nodes $n_{1}, \ldots, n_{N}$ of $\gamma$, in the sense that

$$
\psi\left(\varphi, a^{-1} A a+a^{-1} d a\right)=f\left(\varphi, A, a\left(n_{1}\right), \ldots, a\left(n_{N}\right)\right) .
$$

Therefore,

$$
\int \prod_{x \in \Sigma} d a(x) \psi\left(\varphi, a^{-1} A a+a^{-1} d a\right)=\int d a\left(n_{1}\right) \ldots d a\left(n_{N}\right) \psi\left(\varphi, a^{-1} A a+a^{-1} d a\right)
$$

is actually well defined. This definition of solutions to the Gauss constraint admits interesting generalization to the vector constraint.

Before solving the quantum vector constraint, we decompose the Hilbert space suitably. To every finite set of points

$$
X=\left\{x_{1}, \ldots, x_{k}\right\} \subset \Sigma,
$$

there is naturally assigned a subspace spanned by the states $|\pi\rangle \in \mathscr{H}_{\text {kin,mat }}$ such that the support of the function $\pi: \Sigma \rightarrow \mathbb{R}$ is exactly $X$

$$
\mathscr{D}_{X}=\operatorname{Span}\left(|\pi\rangle \in \mathscr{H}_{\text {kin,mat }}: \operatorname{supp}(\pi)=X\right) \text {. }
$$

The polymer Hilbert space $\mathscr{H}_{\text {kin,mat }}$ is the completion of an orthonormal sum of those subspaces

$$
\mathscr{H}_{\text {kin,mat }}=\bigoplus_{X \subset \Sigma:|X|<\infty} \mathscr{D}_{X} .
$$

We will be precise about the domains of introduced maps, therefore we distinguish here explicitly between the span or infinite direct sum and the completion thereof.

The Hilbert space $\mathscr{H}_{\text {kin,gr }}^{G}$ of the gravitational degrees of freedom is also decomposed into orthogonal subspaces labeled by admissible graphs embedded in $\Sigma$ (see the end of Section 3.3.1 of [23])

$$
\mathscr{H}_{\text {kin,gr }}^{G}=\overline{\bigoplus_{\gamma} \mathscr{D}_{\gamma}^{\prime G}}
$$


where $\gamma$ runs through the set of embedded graphs in $\Sigma$ admissible in the sense, that do not contain any 2 -valent node that can be obtained splitting a single link and possibly reorienting the resulting new links, and

$$
\mathscr{D}_{\gamma}^{\prime G}=\bigoplus_{\vec{j}} \mathscr{H}_{\gamma, \vec{j}, \vec{l}=0}
$$

where each $\vec{j}$ is a coloring of the links by irreducible non-trivial representations of SU(2). The labeling $\vec{l}$, in general case, labels the nodes of $\gamma$ by irreducible representations of SU(2), in this case it is the trivial representation. The sum includes the empty graph $\emptyset$. A cylindrical function with respect to the empty graph is a constant function.

The two decompositions are combined into the decomposition of the total Hilbert space

$$
\mathscr{H}_{\text {kin }}^{G}=\overline{\bigoplus_{(X, \gamma)} \mathscr{D}_{X} \otimes \mathscr{D}_{\gamma}^{\prime G}}
$$

The uncompleted space

$$
\mathscr{D}_{\text {kin }}^{G}:=\bigoplus_{(X, \gamma)} \mathscr{D}_{X} \otimes \mathscr{D}_{\gamma}^{\prime G}
$$

will be an important domain in what follows.

\subsection{Diffeomorphisms and the vector constraint}

The diffeomorphisms of $\Sigma$ act naturally in $\mathscr{H}_{\text {kin }}$,

$$
\operatorname{Diff} \ni \phi \mapsto U(\phi) \in U\left(\mathscr{H}_{\text {kin }}\right),
$$

as the kinematical quantum states are functions of $A$ and $\phi$,

$$
U(\phi) \psi(\varphi, A)=\psi\left(\phi^{*} \varphi, \phi^{*} A\right) .
$$

The only diffeomorphism invariant element of $\mathscr{H}_{\text {kin }}$ is

$$
\psi(\varphi, A)=\text { const. }
$$

However, the analogous to (3.4) averaging with respect to the diffeomorphisms produces a larger than 1-dimensional Hilbert space, containing also "non-normalizable" states. They become normalizable with respect to a natural Hilbert product. The diffeomorphism averaging in the matter free case is discussed in detail in [23]. Now we need to discuss it more closely in the case with the scalar field.

For each of the subspaces $\mathscr{D}_{X} \otimes \mathscr{D}_{\gamma}^{\prime G}$ introduced above, denote by $\operatorname{TDiff}_{X, \gamma}$ the set of the diffeomorphisms which act trivially in $\mathscr{D}_{X} \otimes \mathscr{D}_{\gamma}^{\prime G}$. It is easy to see that $\mathrm{TDiff}_{X, \gamma}$ consists of diffeomorphisms $\phi$ such that

$$
\left.\phi\right|_{X}=\mathrm{id}, \text { and } \phi(\ell)=\ell \text { for every link } \ell \text { of } \gamma,
$$

where we recall that the links are oriented, and the orientation has to be preserved as well. We will average with respect to the group of orbits

$$
\operatorname{Diff} / \operatorname{TDiff}_{X, \gamma} .
$$


Given $\psi \in \mathscr{D}_{X} \otimes \mathscr{D}_{\gamma}^{\prime G}$, what is averaged is the dual state $\langle\psi|$, that is the linear functional on $\mathscr{H}_{\text {kin }}^{\mathrm{G}}$,

$$
\langle\psi|: \psi^{\prime} \mapsto\left(\psi \mid \psi^{\prime}\right)_{\text {kin }}
$$

The averaging formula is simple:

$$
\mathscr{D}_{X} \otimes \mathscr{D}_{\gamma}^{\prime G} \ni \psi \mapsto\langle\psi| \mapsto \frac{1}{n_{X, \gamma}} \sum_{[\phi] \in \operatorname{Diff} / \operatorname{TDiff}_{X, \gamma}}\langle U(\phi) \psi|=: \eta(\psi),
$$

where the factor $\frac{1}{n_{X, \gamma}}$ will be fixed below. The result of the averaging is a linear functional

$$
[\eta(\psi)]\left(\psi^{\prime}\right)=\frac{1}{n_{X, \gamma}} \sum_{[\phi] \in \operatorname{Diff}_{/ \operatorname{Tiff}_{X, \gamma}}}\left(U(\phi) \psi \mid \psi^{\prime}\right)_{\mathrm{kin}} .
$$

Indeed, given $\psi$ and $\psi^{\prime}$ the sum on the RHS contains only finite number of nonzero terms, therefore the formula defines a linear functional $\eta(\psi)$. This functional is invariant with respect to the action of the diffeomorphisms in $\mathscr{H}_{\text {kin }}$. The map $\eta$ is defined for all the subspaces $\mathscr{D}_{X} \otimes \mathscr{D}_{\gamma}^{\prime G}$ and extended by the linearity to their orthogonal sum $\mathscr{D}_{\text {kin }}^{G}$. As in the matter free case [23], one can also consider the subgroup Diff ${ }_{X, \gamma}$ of the diffemorphisms preserving the subspace $\mathscr{D}_{X} \otimes \mathscr{D}_{\gamma}^{\prime G}$. It gives rise to the symmetry group

$$
\mathrm{GS}_{X, \gamma}:=\operatorname{Diff}_{X, \gamma} / \operatorname{TDiff}_{X, \gamma}
$$

which is finite. We fix the number $n_{X, \gamma}$ in the definition of $\eta$ to be

$$
n_{X, \gamma}:=\left|\mathrm{GS}_{X, \gamma}\right|<\infty .
$$

The image of $\eta$ will be denoted as follows

$$
\mathscr{D}_{\text {Diff }}^{G}:=\eta\left(\mathscr{D}_{\text {kin }}^{G}\right)
$$

The new scalar product in $\mathscr{D}_{\text {Diff }}^{G}$ is

$$
\left(\eta(\psi) \mid \eta\left(\psi^{\prime}\right)\right)_{\text {Diff }}:=[\eta(\psi)]\left(\psi^{\prime}\right) .
$$

This completes the construction of the Hilbert space of the solutions to the vector constraint

$$
\mathscr{H}_{\text {Diff }}^{G}=\overline{\eta\left(\mathscr{D}_{\text {kin }}^{G}\right)} .
$$

With this choice of $n_{X, \gamma}$, the map $\eta$ projects $\psi$ orthogonally onto the subspace of $\mathscr{D}_{X} \otimes \mathscr{D}_{\gamma}^{\prime G}$ consisting of the elements symmetric with respect to the symmetries of $(X, \gamma)$, and next unitarily embeds in $\mathscr{H}_{\text {Diff }}^{G}$. More generally, for

$$
\psi_{I} \in \mathscr{D}_{X_{I}} \otimes \mathscr{D}_{\gamma_{I}}^{\prime G}, \quad I=1,2
$$

the scalar product can be written in the following way

$$
\left(\eta\left(\psi_{1}\right) \mid \eta\left(\psi_{2}\right)\right)_{\text {Diff }}=\delta_{\left[X_{1}, \gamma_{1}\right],\left[X_{2}, \gamma_{2}\right]}\left(\psi_{1} \mid P_{X_{2}, \gamma_{2}} \psi_{2}\right)_{\mathrm{kin}},
$$

where: $[X, \gamma]$ is the class of pairs $\left(X^{\prime}, \gamma^{\prime}\right)$ diffeomorphically equivalent to a given pair $(X, \gamma)$, $\delta_{\left[X_{1}, \gamma_{1}\right],\left[X_{2}, \gamma_{2}\right]}$ stands for the Kronecker delta, and

$$
P_{X_{2}, \gamma_{2}}: \overline{\mathscr{D}_{X_{2}} \otimes \mathscr{D}_{\gamma_{2}}^{\prime}{ }^{G}} \rightarrow \overline{\mathscr{D}_{X_{2}} \otimes \mathscr{D}_{\gamma_{2}}^{\prime G}}
$$


is the orthogonal projection onto the subspace of states symmetric with respect to the group $\mathrm{GS}_{X_{2}, \gamma_{2}}$.

The space $\mathscr{H}_{\text {Diff }}^{G}$ is our Hilbert space of solutions to the quantum Gauss and quantum diffeomorphisms constraints. On the other hand, each element of $\mathscr{H}_{\text {Diff }}^{G}$ is a linear functional defined on $\mathscr{D}_{\text {kin }}^{G}$,

$$
\mathscr{H}_{\text {Diff }}^{G} \subset\left(\mathscr{D}_{\text {kin }}^{G}\right)^{*}
$$

where the right hand side is the space of the linear functionals $\mathscr{D}_{\text {kin }}^{G} \rightarrow \mathbb{C}$. We still use that extra structure intensively. In particular, an operator

$$
\hat{\mathscr{O}}: \mathscr{D}_{\text {kin }}^{G} \rightarrow \mathscr{D}_{\text {kin }}^{G}
$$

will be pulled back to the dual operator

$$
\hat{\mathscr{O}}^{*}: \mathscr{D}_{\text {Diff }}^{G} \rightarrow\left(\mathscr{D}_{\text {kin }}^{G}\right)^{*} .
$$

\subsection{The scalar constraint}

In the Hilbert space $\mathscr{H}_{\mathrm{Diff}}^{G}$ of solutions of the quantum Gauss and vector constraint, we impose the quantum scalar constraint

$$
\left(\hat{\pi}(x)^{*}-\hat{h}(x)\right) \Psi=0 .
$$

In $[13,23]$ it is argued that a general solution can be derived if one is able to introduce an operator

$$
\exp i \int d^{3} x \hat{\varphi}(x) \hat{h}(x): \mathscr{H}_{\text {Diff }}^{G} \rightarrow \mathscr{H}_{\text {Diff }}^{G}
$$

of suitable, but quite natural, properties. We will define now such an operator in the very space $\mathscr{H}_{\text {Diff }}^{G}$ and see that it does have the desired properties.

\subsubsection{Extra structure needed for $\hat{h}(x)$}

To deal with the operator (distribution) $\hat{h}(x)$ we will need more structure. For each graph $\gamma$ its set of nodes will be denoted by $\gamma^{0}$. For every of the subspaces $\mathscr{D}_{X} \otimes \mathscr{D}_{\gamma}^{\prime G}$ (modulo the diffeomorphisms) it is convenient to consider the subgroup

$$
\operatorname{Diff}_{X \cup \gamma^{0}}
$$

of Diff set by the diffeomorphisms which act as identity on the set $X$ as well as on the set $\gamma^{0}$ of the nodes of $\gamma$. We repeat the construction of the averaging for the diffeomorphisms Diff $\operatorname{D}_{X \gamma^{0}}$,

$$
\mathscr{D}_{X} \otimes \mathscr{D}_{\gamma}^{\prime G} \ni \psi \mapsto \tilde{\eta}(\psi)=\frac{1}{\tilde{n}_{X, \gamma}} \sum_{[\phi] \in \operatorname{Diff}_{\mathrm{X} \cup \gamma} / \operatorname{TDiff}_{X, \gamma}}\langle U(\phi) \psi|
$$

where the number $\tilde{n}_{X, \gamma}$ will be fixed later to be consistent with another map $\check{\eta}$ introduced below. For example, if $\psi \in \mathscr{D}_{X} \otimes \mathscr{D}_{\gamma}^{\prime G}$ is a simple tensor product

$$
\psi=\langle\pi| \otimes f_{\gamma}
$$

then,

$$
\tilde{\eta}(\psi)=\frac{\langle\pi|}{\tilde{n}_{X, \gamma}} \otimes \sum_{[\phi] \in \operatorname{Diff}_{\mathrm{X} \cup \gamma^{0}} / \operatorname{TDiff}_{X, \gamma}}\left\langle U(\phi) f_{\gamma}\right| .
$$


Given a finite set $Y \subset \Sigma$, we consider all the spaces $\mathscr{D}_{X} \otimes \mathscr{D}_{\gamma}$ such that

$$
X \cup \gamma^{0}=Y,
$$

combine them into the space

$$
\bigoplus_{(X, \gamma)} \mathscr{D}_{X} \otimes \mathscr{D}_{\gamma}^{\prime G}
$$

and combine the maps $\tilde{\eta}$ to a linear map

$$
\tilde{\eta}: \bigoplus_{(X, \gamma)} \mathscr{D}_{X} \otimes \mathscr{D}_{\gamma}^{\prime G} \rightarrow\left(\mathscr{D}_{\mathrm{kin}}^{\prime G}\right)^{*}
$$

and endow the image of this map

$$
\mathscr{D}_{\mathrm{Diff}_{Y}}^{G}:=\tilde{\eta}\left(\bigoplus_{(X, \gamma)} \mathscr{D}_{X} \otimes \mathscr{D}_{\gamma}^{\prime G}\right),
$$

with a scalar product

$$
\left(\tilde{\eta}\left(\psi_{1}\right) \mid \eta\left(\tilde{\psi}_{2}\right)\right)_{\operatorname{Diff}}:=\left[\tilde{\eta}\left(\psi_{1}\right)\right]\left(\psi_{2}\right) .
$$

In this way we obtain the Hilbert space

$$
\mathscr{H}_{\mathrm{Diff}_{Y}}^{G}=\overline{\mathscr{D}_{\mathrm{Diff}_{Y}}^{G}},
$$

that is needed to deal with the $\hat{h}(x)$ operator.

The original averaging map $\eta$ for $\psi \in \mathscr{D}_{X} \otimes \mathscr{D}_{\gamma}^{G}$ can be written as averaged $\tilde{\eta}$,

$$
\eta(\psi)=\frac{1}{|Y| !} \sum_{[\phi] \in \operatorname{Diff}_{/} \operatorname{Diff}_{Y}} U(\phi)^{*} \tilde{\eta}(\psi),
$$

where the choice of the normalization factor as the number of the elements of the symmetry group of the set $Y$ is the condition that fixes the number $\tilde{n}_{X, \gamma}$ of (3.23) uniquely. The map $\tilde{\eta}(\psi) \mapsto \eta(\psi)$ extends by the continuity to

$$
\mathscr{H}_{\mathrm{Diff} Y}^{G} \rightarrow \mathscr{H}_{\mathrm{Diff}}^{G}, \quad \check{\eta}(\tilde{\Psi})=\frac{1}{|Y| !} \sum_{[\phi] \in \operatorname{Diff}^{\prime} \operatorname{Diff}_{Y}} U(\phi)^{*} \tilde{\Psi} .
$$

The factor $|Y|$ ! ensures, that for every $\tilde{\Psi}_{I}, I=1,2$ invariant with respect to all $\operatorname{Diff} Y$,

$$
\left(\check{\eta}\left(\tilde{\Psi}_{I}\right) \mid \check{\eta}\left(\tilde{\Psi}_{J}\right)\right)_{\operatorname{Diff}}=\left(\tilde{\Psi}_{I} \mid \tilde{\Psi}_{J}\right)_{\operatorname{Diff}_{Y}} .
$$

Before we apply this structure to the operator $\hat{h}(x)$, let us use it to characterize the action of the operator $\hat{\pi}(x)^{*}$ defined by the duality on the diffeomorphism invariant states, elements of the space $\mathscr{D}_{\text {diff }}^{G} \subset\left(\mathscr{D}_{\text {kin }}^{G}\right)^{*}$. It will be convenient to introduce for each $y \in \Sigma$, an operator $\hat{\pi}_{y}$ defined in (a suitable domain of) $\mathscr{H}_{\text {kin,mat }}$ by $\hat{\pi}(x)$,

$$
\hat{\pi}(x)=\sum_{y \in \Sigma} \delta(x, y) \hat{\pi}_{y}, \quad \hat{\pi}_{y}|\pi\rangle=\pi_{y}|\pi\rangle,
$$


(recall that given $|\pi\rangle$, the function $y \mapsto \pi_{y}$ is not zero only for a finite set of points $y$ ). This definition passes by the duality to the (bra) states corresponding to the (ket) states $|\pi\rangle$ via $\langle\pi|\left(\left|\pi^{\prime}\right\rangle\right):=$ $\left(\pi \mid \pi^{\prime}\right)_{\text {kin }}$

$$
\hat{\pi}_{y}^{*}\langle\pi|=\pi_{y}\langle\pi|
$$

Next, increasing the level of complexity, consider the action of the operator $\hat{\pi}_{y}^{*}$ in each of the spaces $\mathscr{D}_{\text {Diff }}^{G}$. To begin with

$$
\left.y \notin Y \Rightarrow \hat{\pi}_{y}^{*}\right|_{\mathscr{D}_{\operatorname{Diff}_{Y}}^{G}}=0 .
$$

The elements $\tilde{\eta}\left(\langle\pi| \otimes f_{\gamma}\right)$ are eigenvectors,

$$
\hat{\pi}_{y}^{*} \tilde{\eta}\left(\langle\pi| \otimes f_{\gamma}\right)=\pi_{y} \tilde{\eta}\left(\langle\pi| \otimes f_{\gamma}\right) .
$$

Finally, to write the action of $\hat{p i} i_{y}^{*}$ in $\mathscr{H}_{\text {Diff }}^{G}$, given

$$
\tilde{\Psi} \in \mathscr{D}_{\text {DiffY }}^{G}, \quad \text { and } \quad \check{\eta}(\tilde{\Psi}) \in \mathscr{H}_{\text {Diff }}^{G}
$$

we have

$$
\hat{\pi}_{y}^{*} \check{\eta}(\tilde{\Psi})=\frac{1}{|Y| !} \sum_{y^{\prime} \in Y} \sum_{\left[\phi_{y^{\prime}}\right]} \hat{\pi}_{y}^{*} U\left(\phi_{y^{\prime}}\right)^{*} \tilde{\psi}=\frac{1}{|Y| !} \sum_{y^{\prime} \in Y} \sum_{\left[\phi_{y^{\prime}}\right]} U\left(\phi_{y^{\prime}}\right)^{*} \hat{\pi}_{y^{\prime}}^{*} \tilde{\psi}
$$

where for every $y^{\prime} \in Y,\left[\phi_{y^{\prime}}\right]$ runs through the subset of Diff/Diff $Y_{Y}$ such that

$$
\phi_{y^{\prime}}(y)=y^{\prime}
$$

For

$$
\tilde{\Psi}=\langle\pi| \otimes \tilde{f},
$$

we have

$$
\hat{\pi}_{y}^{*} \check{\eta}(\langle\pi| \otimes \tilde{f})=\frac{1}{|Y| !} \sum_{y^{\prime} \in Y} \pi_{y^{\prime}} \sum_{\left[\phi_{y^{\prime}}\right]} U\left(\phi_{y^{\prime}}\right)^{*}\langle\pi| \otimes \tilde{f} .
$$

The result of the action is not any longer an element of $\mathscr{H}_{\text {diff }}^{G}$, however the operator $\hat{\pi}_{y}$ is well defined in the domain $\mathscr{D}_{\text {diff }}^{G} \subset \mathscr{H}_{\text {diff }}^{G}$ in the following sense

$$
\hat{\pi}_{y}: \mathscr{D}_{\text {diff }}^{G} \rightarrow\left(\mathscr{D}_{\text {kin }}^{G}\right)^{*} .
$$

Now, we are in the position to write down the action of the operator $\hat{h}(x)$ apparent in the quantum scalar constraint. It is not defined directly in $\mathscr{H}_{\text {kin,gr }}$, however it is defined in the spaces $\mathscr{H}_{\text {Diffy }}^{G}$. Actually, it is introduced in the opposite order [13,23] than the calculation of the action of $\hat{\pi}(x)$ was performed above.

First, in each of the spaces $\mathscr{H}_{\mathrm{Diff}_{Y}}^{G}$ and for every $y \in \Sigma$ the operator $\hat{h}_{y}$ is defined as a self-adjoint operator. The operator is identically zero unless $y \in Y$,

$$
\left.y \notin Y \Rightarrow \hat{h}_{y}\right|_{\mathscr{H}_{\operatorname{Difff}_{Y}^{G}}^{G}}=0 .
$$

By the linearity, $\hat{h}_{y}$ is extended to the span

$$
\operatorname{Span}\left(\mathscr{H}_{\mathrm{Diff}_{Y}}^{G}: Y \subset \Sigma,|Y|<\infty\right) \subset\left(\mathscr{D}_{\text {kin }}^{G}\right)^{*} .
$$


For different points the operators commute,

$$
y \neq y^{\prime} \Rightarrow\left[\hat{h}_{y}, h_{y^{\prime}}\right]=0 .
$$

The map $y \mapsto \hat{h}_{y}$ is diffeomorphism invariant in the sense that for every diffeomorphism $\phi \in$ Diff and its (dual) action $U(\phi)^{*}$ in the subset (3.30) of $\left(\mathscr{D}_{\text {kin }}^{G}\right)^{*}$ we have

$$
\hat{h}_{\phi^{-1}(y)} U(\phi)^{*}=U(\phi)^{*} \hat{h}_{y} .
$$

The action of $\hat{h}_{y}$ is $\mathscr{H}_{\text {Diff }}^{G}$ is defined by the analogy to (3.28), that is given

$$
\tilde{\Psi} \in \mathscr{D}_{\text {DiffY }}^{G}, \quad \text { and } \quad \check{\eta}(\tilde{\Psi}) \in \mathscr{H}_{\text {Diff }}^{G}
$$

we have

$$
\hat{h}_{y} \check{\eta}(\tilde{\Psi})=\frac{1}{|Y| !} \sum_{y^{\prime} \in Y} \sum_{\left[\phi_{y^{\prime}}\right]} \hat{h}_{y} U\left(\phi_{y^{\prime}}\right)^{*} \tilde{\psi}=\frac{1}{|Y| !} \sum_{y^{\prime} \in Y} \sum_{\left[\phi_{y^{\prime}}\right]} U\left(\phi_{y^{\prime}}\right)^{*} \hat{h}_{y^{\prime}} \tilde{\psi}
$$

where the notation is the same as in (3.28)

\subsubsection{The $\exp \left(\int d^{3} x \hat{\varphi}(x) \hat{h}(x)\right)$ operator}

We can turn now, to the introduction of an operator $\exp \left(i \int d^{3} x \hat{\varphi}(x) \hat{h}(x)\right)$. For every of the spaces $\mathscr{H}_{\mathrm{Diff}_{Y}}^{G}$ there is a basis of simultaneous eigenvectors of the operators $\hat{h}_{y}$ and $\hat{\pi}_{y}, y \in \Sigma$. We choose a one, and denote its elements by $\langle\pi| \otimes\langle h, \alpha|$ where

$$
h: y \mapsto h_{y}, \quad \pi: y \mapsto \pi_{y}
$$

are functions of finite supports such that

$$
\hat{h}_{y}\langle\pi| \otimes\langle h, \alpha|=h_{y}\langle\pi| \otimes\langle h, \alpha|, \quad \hat{\pi}_{y}\langle\pi| \otimes\langle h, \alpha|=\pi_{y}\langle\pi| \otimes\langle h, \alpha|
$$

and $\alpha$ is an extra label. We define (compare with (2.4))

$$
e^{i \int d^{3} x \hat{\varphi}(x) \hat{h}(x)}\langle\pi| \otimes\langle h, \alpha|=\langle\pi+h| \otimes\langle h, \alpha| .
$$

That defines an operator in each of the spaces $\mathscr{H}_{\text {Diff }}$ and in the span which is the direct (orthogonal) $\operatorname{sum}(3.30)$

This operator is unitary,

$$
\left(e^{i \int d^{3} x \hat{\varphi}(x) \hat{h}(x)}\right)^{\dagger}=e^{-i \int d^{3} x \hat{\varphi}(x) \hat{h}(x)},
$$

where the right hand side is defined by

$$
e^{-i \int d^{3} x \hat{\varphi}(x) \hat{h}(x)}\langle\pi| \otimes\langle h, \alpha|=\langle\pi-h| \otimes\langle h, \alpha| .
$$

The operator is diffeomorphisms invariant,

$$
U(\phi)^{*} e^{i \int d^{3} x \varphi \hat{(x)} \hat{h}(x)}=e^{i \int d^{3} x \hat{\varphi}(x) \hat{h}(x)} U(\phi)^{*} .
$$


Finally, to define this operator in $\mathscr{H}_{\text {Diff }}^{G}$, for every $\tilde{\Psi} \in \mathscr{H}_{\text {Diff }}$ and the corresponding $\check{\eta}(\tilde{\Psi}) \in$ $\mathscr{H}_{\text {Diff }}^{G}$ we write

$$
e^{i \int d^{3} x \hat{\varphi}(x) \hat{h}(x)} \check{\eta}(\tilde{\Psi}):=\frac{1}{|Y|} \sum_{[\phi] \in \operatorname{Diff} / \operatorname{Diff}_{Y}} e^{i \int d^{3} x \hat{\varphi}(x) \hat{h}(x)} U(\phi)^{*} \tilde{\Psi}
$$

Indeed, we can always do it, but is the right hand side again an element of the Hilbert space $\mathscr{H}_{\text {Diff }}^{G}$ ? The answer is affirmative due to the diffeomorphism invariance, namely, it follows that

$$
e^{i \int d^{3} x \hat{\varphi}(x) \hat{h}(x)} \check{\eta}(\tilde{\Psi})=\check{\eta}\left(e^{i \int d^{3} x \hat{\varphi}(x) \hat{h}(x)} \tilde{\Psi}\right) \in \mathscr{H}_{\text {Diff }}^{G} .
$$

The extension by the linearity and continuity provides a unitary operator

$$
e^{i \int d^{3} x \hat{\varphi}(x) \hat{h}(x)}: \mathscr{H}_{\text {Diff }}^{G} \rightarrow \mathscr{H}_{\text {Diff }}^{G}
$$

for which the property (3.33) still holds.

Now, it is not hard to check, that our operator (3.36) does satisfy the desired property, namely for every $\Psi \in \mathscr{H}_{\text {Diff, }}^{G}$,

$$
e^{-i \int d^{3} x \hat{\varphi}(x) \hat{h}(x)}(\hat{\pi}(y)-\hat{h}(y)) e^{i \int d^{3} x \hat{\varphi}(x) \hat{h}(x)} \Psi=\hat{\pi}(y) \Psi \in\left(\mathscr{D}_{\text {Diff }}^{G}\right)^{*} .
$$

\subsubsection{Solutions, Dirac observables, dynamics}

The quantum scalar constraint

$$
(\hat{\pi}(x)-\hat{h}(x)) \Psi=0
$$

is equivalent to

$$
\hat{\pi}(x) e^{-i \int d^{3} x \hat{\varphi}(x) \hat{h}(x)} \Psi=0 .
$$

Moreover, the condition on the Dirac observable

$$
[\hat{\pi}(x)-\hat{h}(x), \hat{\mathscr{O}}]=0
$$

is equivalent to

$$
\left[\hat{\pi}(x), e^{-i \int d^{3} x \hat{\varphi}(x) \hat{h}(x)} \hat{\mathscr{O}} e^{i \int d^{3} x \hat{\varphi}(x) \hat{h}(x)}\right]=0 .
$$

In $\mathscr{H}_{\text {Diff }}^{G}$, solutions to the equation

$$
\hat{\pi}(x) \Psi^{\prime}=0
$$

set the subspace given by

$$
\overline{\eta\left(|0\rangle \otimes \bigoplus_{\gamma} \mathscr{D}_{\gamma}^{\prime G}\right)}=\mathscr{H}_{\text {Diff,gr }}^{G},
$$

that is the subspace of states independent of $\varphi$. Hence, solutions to the quantum scalar (and the Gauss) constraint are

$$
\mathscr{H}_{\text {Diff }}^{G} \ni \Psi=e^{i \int d^{3} x \hat{\varphi}(x) \hat{h}(x)} \Psi^{\prime}, \quad \Psi^{\prime} \in \mathscr{H}_{\text {Diff,gr }}^{G} .
$$


Denote the subspace they set by

$$
\mathscr{H}_{\text {phys }} \subset \mathscr{H}_{\text {Diff }}^{G}
$$

A Dirac observable is every operator

$$
e^{i \int d^{3} x \hat{\varphi}(x) \hat{h}(x)} \hat{L} e^{-i \int d^{3} x \hat{\varphi}(x) \hat{h}(x)}
$$

defined in $\mathscr{H}_{\text {phys }}$ by an operator $\hat{L}$ defined in $\mathscr{H}_{\text {Diff,gr }}^{G}$. Another observable can be defined from the operators $\hat{\pi}(x)$, for example

$$
\int d^{3} x \hat{\pi}(x)
$$

however,

$$
\left.\hat{\pi}(x)\right|_{\mathscr{H}_{\text {phys }}}=\left.\hat{h}(x)\right|_{\mathscr{H}_{\text {phys }}}
$$

and $\hat{h}(x)$ is defined in $\mathscr{H}_{\text {Diff,gr }}^{G}$. Our map (3.36) can be generalized to a family of maps corresponding to the transformation $\phi \mapsto \phi+\tau, \tau \in \mathbb{R}$. For every $\tau$ the transformation should amount to a transformation

$$
e^{i \int d^{3} x \tau \hat{h}(x)}: \mathscr{H}_{\text {Diff }}^{G} \rightarrow \mathscr{H}_{\text {Diff }}^{G},
$$

where the operator has to be defined. To define the operator $\exp \left(i \int d^{3} x \tau \hat{h}(x)\right)$ we repeat the construction that lead us to the operator $\exp \left(i \int d^{3} x \hat{\varphi} \hat{h}(x)\right)$, with the starting point

$$
e^{i \int d^{3} x \hat{h}(x)}\langle\pi| \otimes\left\langle h, X, \gamma^{0}, \alpha\right|=e^{i \sum_{x} h_{x}}\langle\pi| \otimes\left\langle h, X, \gamma^{0}, \alpha\right| .
$$

As expected, the operator preserves the space of solutions

$$
e^{i \int d^{3} x \tau \hat{h}(x)}\left(\mathscr{H}_{\text {phys }}\right)=\mathscr{H}_{\text {phys }}
$$

and defines therein the dynamics.

\section{Summary and seeds of a new idea}

The first conclusion is that a quantization of the scalar field whose existence and suitable properties were assumed in [13] exists, and an example is the polymer quantization. Furthermore, it is shown explicitly, that as argued in [13], the theory is equivalent to the quantum theory in the Hilbert space $\mathscr{H}_{\text {Diff,gr }}^{G}$ of diffeomorphism invariant states of the gravitational degrees of freedom only, with the dynamics defined by the physical Hamiltonian

$$
\hat{h}_{\text {phys }}=\int d^{3} x \hat{h}(x),
$$

where $\hat{h}(x)$ is a quantization of the classical solution for $\pi(x)$

$$
\pi(x)=h(x)
$$

following from the constraints. In this way, the current work completes the derivation of the model already formulated in [13]. Technically, we have implemented in detail the diffeomorphism averaging for loop quantum gravity states of geometry coupled with the polymer states of scalar field 
and discussed the general structure of the operators emerging in the scalar constraints. Mathematically, the physically relevant part of the Hilbert space $\mathscr{H}_{\text {Diff }}^{G}$ is contained in the so called habitat space introduced in [24]. This is a new application of the habitat framework which may be useful for various technical questions.

Secondly, it turns out, that in the framework of the polymer quantization of the scalar field, the Hilbert space $\mathscr{H}_{\text {phys }}$ of the physical states, solutions to the quantum constraints, is a subspace of the Hilbert space of solutions to the diffeomorphism constraint,

$$
\mathscr{H}_{\text {phys }} \subset \mathscr{H}_{\text {Diff }}^{G} \text {. }
$$

Therefore, more structure is at our disposal, than only the physical states themselves. This advantage is not only estetic. It also gives a clue for quite promising development of the theory. We explain this below.

The classical constraints for the massless field coupled to gravity are

$$
\begin{gathered}
C(x)=C^{\mathrm{gr}}(x)+\frac{1}{2} \frac{\pi^{2}(x)}{\sqrt{q(x)}}+\frac{1}{2} q^{a b}(x) \phi_{, a}(x) \phi_{, b}(x) \sqrt{q(x)}, \\
C_{a}(x)=C_{a}^{\mathrm{gr}}(x)+\pi(x) \phi_{, a}(x) .
\end{gathered}
$$

where $q_{a b}$ is the 3-metric tensor induced on a 3-slice of spacetime $C^{\mathrm{gr}}$ is the gravitational field part of the scalar constraint, and $C_{a}^{\text {gr }}$ is the gravitational part of the vector constraint.

The scalar constraint $C(x)$ can be replaced by $C^{\prime}(x)$ (deparametrized scalar constraint):

$$
\begin{gathered}
C^{\prime}(x)=\pi^{2}(x)-h^{2}(x), \\
h_{ \pm}:=\sqrt{-\sqrt{q} C^{\mathrm{gr}}+/-\sqrt{q} \sqrt{\left(C^{\mathrm{gr}}\right)^{2}-q^{a b} C_{a}^{\mathrm{gr}} C_{b}^{\mathrm{gr}}}} .
\end{gathered}
$$

The sign \pm in $h_{ \pm}$is + in the part of the phase space at which

$$
\pi^{2} \geq \phi_{, a} \phi_{, b} q^{a b} \operatorname{det} q
$$

for example in the neighborhood of the homogeneous solutions.

The sign \pm in $h_{ \pm}$is - , on the other hand, in the part of the phase space at which

$$
\pi^{2} \leq \phi_{, a} \phi_{, b} q^{a b} \operatorname{det} q
$$

Each of the cases $(4.6,4.7)$ consists of two in cases,

$$
\pi(x)=+h_{ \pm}(x), \quad \text { or } \quad \pi(x)=-h_{ \pm}(x) .
$$

A natural first goal [13], was to restrict the quantization to the case (4.6) and positive $\pi$, and quantize the theory for the part of the phase space which contains expanding homogeneous solutions. Now, the formulation of the current paper allows an attempt to unify the theory to the both cases (4.6) and (4.7) the both cases (4.8). Indeed, we can accommodate in the Hilbert space $\mathscr{H}_{\text {Diff }}^{G}$ simultaneously quantum solutions to each of the cases. To this end, one has to implement the construction presented in the current paper for each of the following 4 cases

$$
\hat{h}(x)=\hat{h}_{+}(x),-\hat{h}_{+}(x), \hat{h}_{-}(x),-\hat{h}_{-}(x) .
$$


The result will be four subspaces

$$
\mathscr{H}_{\text {phys }++}, \mathscr{H}_{\text {phys-+ }}, \mathscr{H}_{\text {phys }+-}, \mathscr{H}_{\text {phys-- }} \subset \mathscr{H}_{\text {Diff }}^{G} .
$$

They span the total space of solutions

$$
\mathscr{H}_{\text {phys }}=\operatorname{Span}\left(\mathscr{H}_{\text {phys }++}, \mathscr{H}_{\text {phys-+}}, \mathscr{H}_{\text {phys }+-}, \mathscr{H}_{\text {phys }--}\right) \subset \mathscr{H}_{\text {Diff }}^{G}
$$

The space is endowed with the evolution induced by the transformation

$$
\varphi \mapsto \varphi+\tau
$$

Whether this is it, or more input is needed is an open question. In any case. certainly, this framework takes us beyond the state of art.

\section{Acknowledgements}

We benefited a lot from comments of Andrea Dapor, Paweł Duch and Hanno Sahlmann. This work was partially supported by the grant of Polish Ministerstwo Nauki i Szkolnictwa Wyższego nr N N202 104838 and by the grant of Polish Narodowe Centrum Nauki nr 2011/02/A/ST2/00300.

\section{References}

[1] T. Thiemann (2007) Modern Canonical Quantum General Relativity, Cambridge University Press, Cambridge

[2] C. Rovelli (2004) Quantum Gravity, Cambridge University Press, Cambridge

[3] R. Gambini, J. Pullin (1996) Loops, Knots, Gauge Theories and Quantum Gravity, Cambridge University Press, Cambridge

[4] A. Ashtekar, J. Lewandowski (2004) Background independent quantum gravity: a status report, Class. Quantum Grav. 21, R53

[5] A. Ashtekar, J. Lewandowski (2001) Relation between polymer and Fock excitations, Class. Quantum Grav. 18, L117

[6] A. Ashtekar, H. Sahlmann, J. Lewandowski (2003) Polymer and Fock representation for a scalar field, Class. Quantum Grav. 20, L11

[7] T. Thiemann (1998) Kinematical Hilbert spaces for fermionic and Higgs quantum field theories, Class. Quantum Grav. 15, 1487

[8] W. Kamiński, J. Lewandowski, M. Bobieński (2006) Background independent quantizations: the scalar field I, Class. Quantum Grav. 23, 2761

[9] W. Kamiński, J. Lewandowski, A. Okołów (2006) Background independent quantizations: the scalar field II, Class. Quantum Grav. 23, 5547

[10] C. Rovelli, L. Smolin (1993) The physical hamiltonian in non-perturbative quantum gravity, Phys. Rev. Lett. 72, 446

[11] J. D. Brown, K. V. Kuchar (1995) Dust as a standard of space and time in canonical quantum gravity, Phys. Rev. D 51, 5600 
[12] K. Giesel, T. Thiemann (2010) Algebraic quantum gravity (AQG). IV. Reduced phase space quantization of loop quantum gravity, Class. Quantum Grav. 27, 175009

[13] M. Domagała, K. Giesel, W. Kamiński, J. Lewandowski (2010) Gravity quantized, Phys. Rev. D 82, 104038

[14] R. Gambini, J. Pullin (2012) Spherically symmetric gravity coupled to a scalar field with a local Hamiltonian: the complete initial-boundary value problem using metric variables, arXiv:1207.6028

[15] A. Ashtekar, P. Singh (2011) Loop Quantum Cosmology: A Status Report, Class. Quantum Grav. 28, 213001

[16] M. Bojowald (2008) Loop Quantum Cosmology, Living Rev. Relativity 11, 4

[17] A. Ashtekar, J. Lewandowski (2001) Relation between polymer and Fock excitations, Class. Quantum Grav. 18, L117

[18] A. Ashtekar, S. Fairhurst, J. Willis (2002) Quantum gravity, shadow states, and quantum mechanics, Class. Quantum Grav. 20, 1031

[19] K. Fredenhagen, F. Reszewski (2006) Polymer state approximations of Schroedinger wave functions, Class. Quantum Grav. 23, 6577

[20] A. Ashtekar, T. Pawłowski, P. Singh (2006) Quantum Nature of the Big Bang: Improved dynamics, Phys. Rev. D 74, 084003

[21] W. Kamiński, J. Lewandowski (2008) The flat FRW model in LQC: the self-adjointness, Class. Quantum Grav. 25, 035001

[22] A. Ashtekar, M. Bojowald, J. Lewandowski (2003) Mathematical structure of loop quantum cosmology, Adv. Theor. Math. Phys. 7, 233

[23] K. Giesel, H. Sahlmann (2012) From Classical to Quantum Gravity: Introduction to Loop Quantum Gravity, Proceedings of the 3rd Quantum Geometry and Quantum Gravity School in Zakopane, arXiv: 1203.2733

[24] J. Lewandowski, D. Marolf (1998) Loop constraints: A habitat and their algebra, Int. J. Mod. Phys. D7, 299 